\title{
Eccentric rotary swaging variants
}

Svetlana Ortmann Ishkina ${ }^{1, *}$, Anastasiya Toenjes ${ }^{2}$, Christian Schenck ${ }^{1,3}$, Axel von Hehl ${ }^{2,3}$, Hans-Werner Zoch ${ }^{2,3}$, and Bernd Kuhfuss ${ }^{1,3}$

${ }^{1}$ bime - Bremen Institute for Mechanical Engineering, University of Bremen, Faculty of Production Engineering, 28359 Bremen, Germany

${ }^{2}$ Leibniz Institute for Materials Engineering - IWT, University of Bremen, Faculty of Production Engineering, 28359 Bremen, Germany

${ }^{3}$ MAPEX - Center for Materials and Processing, University of Bremen, Faculty of Production Engineering, 28359 Bremen, Germany

Received: 17 December 2018 / Accepted: 24 April 2019

\begin{abstract}
Rotary swaging is an incremental cold forming process that changes beneath the geometry also the microstructure and mechanical properties of the workpiece. Especially a new process design with Eccentric Flat Shaped Dies (EFSD) influences both the kind and amount of stress and plastic strain and consequently the material structure, and hence the material and workpiece properties. Eccentric rotary swaging typically provides a helical material flow. According to the process parameters the microstructure features a typical eddy pattern with a spiral shaped grain orientation. The forming process can be carried out in one or more process steps. In a multistage process, it is possible to change the feed direction and, hence, the material flow helix direction. This approach can be used as a possibility to improve the homogeneity of the workpiece and material properties. In addition, for this aims an intermediate heat treatment in multistage forming operations could be realized. Following the goal of optimizing the final properties, the question arises how these mechanical and thermal treatments affect the material microstructure and the forming properties of the workpiece and how they interact. Experiments were conducted with austenitic stainless steel rods of grade AISI304. The effects of the varied feed direction, feed velocity and heat treatment between the forming operations are discussed.
\end{abstract}

Keywords: Cold forming / microstructure / metal forming

\section{Introduction}

Metastable austenitic steels are characterized by an elevated phase hardening due to strain-induced transformation of austenite into martensite especially by multistage processing. An improvement of material characteristics and a better formability of parts are typical for ultra-fine grained (UFG) materials that are characterized by good strength in combination with sufficient ductility [1,2]. Changing of material properties, i.e. decrease of the strain and increase of the ultimate tensile strength more than twice compared to the initial values, could be achieved by cold forming processes like rotary swaging [3]. In this process, the workpiece is fed axially into the swaging head with a feed velocity $v_{\mathrm{f}}$. The tools revolve around the workpiece and oscillate radially with a small amplitude $h_{\mathrm{T}}$, see Figure 1. By means of the movable wedges the closing pressure and the final diameter can be adjusted. Swaged parts are well used in automobile

\footnotetext{
* e-mail: ishkina@bime.de
}

industry due to the dynamics and stress capacity, for example steering shafts. The variation of the process parameter during rotary swaging leads to changing of the mechanical properties and of the swaged parts [4]. It can be used at preparation of semi-finished products with both a calibrated diameter as well as predefined material properties of the parts. To modify the microstructure of swaged rods with small diameter about $1 \mathrm{~mm}$ an approach of eccentric rotary swaging was applied [5]. The eccentric machined parts featured very fine grains. For these components, besides the phase hardening, a work softening could also be detected. Such material characteristics provide advantages for downstream forming processes like for example extrusion [6]. Nevertheless, the eccentric swaged parts had an inhomogeneous structure with well distinguishable grains in a core area and a non-defined structure with spiral shaped orientation in a boundary area [5].

Also, subsequent heat treatment influences the microstructure. During cold forming like rotary swaging, the formability of the metal generally decreases with increasing forming ratio. To regain formability of the material for 


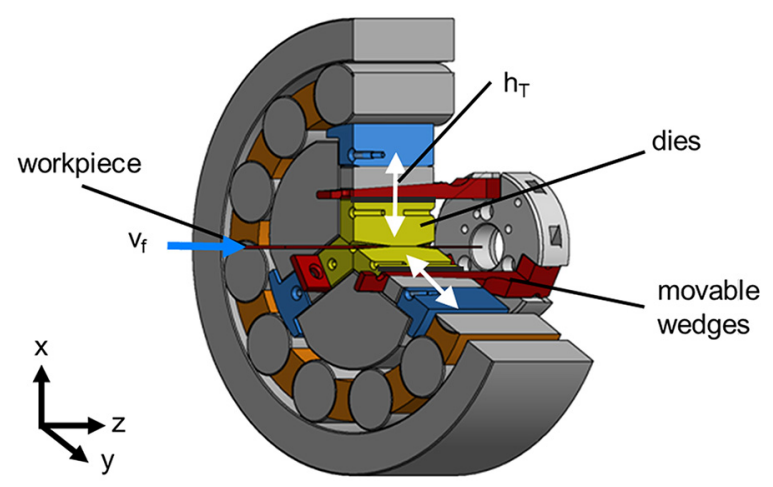

Fig. 1. Rotary swaging head.

Table 1. Chemical composition of stainless steel 1.4301 (AISI304) according to melt analysis.

\begin{tabular}{|c|c|c|c|c|c|c|}
\hline & $\mathrm{C}$ & $\mathrm{Si}$ & $\mathrm{Mn}$ & $\mathrm{P}$ & $\mathrm{Cr}$ & $\mathrm{Ni}$ \\
\hline Ма.-\% & 0.018 & 0.50 & 1.00 & 0.027 & 18.19 & 10.44 \\
\hline
\end{tabular}

further forming steps, a heat treatment process like recrystallization annealing is often necessary in industrial applications. The energy that was consumed by the material during swaging is the driving force for the grain growth and dislocation movement during recrystallization [7]. The aim of this study is to analyze the evolution of the microstructure and the mechanical properties after modification of the eccentric rotary swaging by means of incremental two steps process with change of feed direction and intermediate heat treatment. A disadvantage of eccentric rotary swaging was found as deep cracks on the surface [8]. This problem was faced with special swaging tools (Eccentric Flat Shaped Dies - EFSD), which follow the same kinematic principle like eccentric swaging [9]. The influence of these dies on the boundary area of the workpiece transverse section is discussed in the present work. The microstructure of a variety of eccentrically swaged components is investigated microscopically. The microhardness was obtained for the transverse section of samples as well as for the longitudinal section. The influence of the intermediate heat treatment between two swaging steps on the microstructure and material properties was analyzed. The influence of eccentric rotary swaging variants on the homogeneity, material properties and the surface of the components was studied.

\section{Experiments}

For the experimental tests, sections from the austenitic stainless steel 1.4301 (AISI304) with an initial diameter of $d_{0}=2.7 \mathrm{~mm}$ and a length of $l_{0}=110 \mathrm{~mm}$ were used. The chemical composition of the material is shown in Table 1 and the microstructure before forming is shown in Figure 2.

Experiments were conducted in a rotary swaging machine using three EFSDs (Fig. 3). The workpieces were fed into the swaging head with a constant velocity of

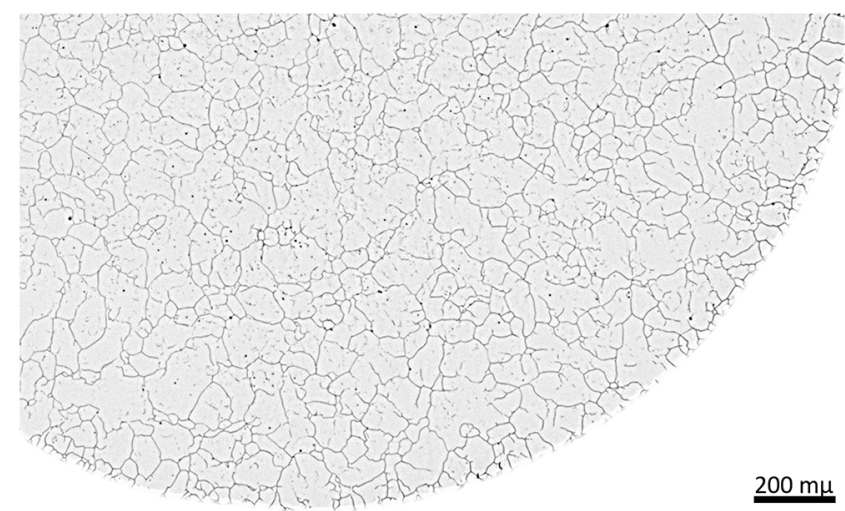

Fig. 2. Transverse section of the microstructure of stainless steel 1.4301 before forming. (a)

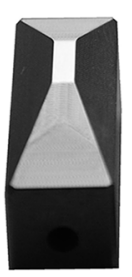

(b)

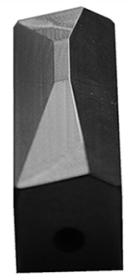

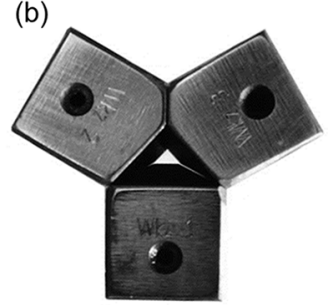

Fig. 3. Eccentric flat shaped dies: (a) single tools and (b) tool assembly.

$v_{\mathrm{f}}=1.0 \mathrm{~mm} / \mathrm{s}$ or $v_{\mathrm{f}}=2.0 \mathrm{~mm} / \mathrm{s}$. The strokes of all swaging dies were equal to $h_{\mathrm{T}}=0.4 \mathrm{~mm}$ and the stroke frequency was kept constant at $f_{\text {st }}=102 \mathrm{~Hz}$ for each experiment.

All samples were formed in two steps. After the first step, the feed direction was converted and the second forming step was performed. In one part of the experiments, the feed velocities were also varied between two steps. In addition, a second series of experiments with the same forming parameters was investigated in which a heat treatment at $600^{\circ} \mathrm{C}$ for $1 \mathrm{~h}$ using a vacuum furnace (Producer ALD, Type VKNQ) was carried out between step 1 and step 2 . The forming parameters are summarized in Table 2.

For comparison, an additional experiment series with a constant velocity of $v_{\mathrm{f}}=1.0 \mathrm{~mm} / \mathrm{s}$ (setting 1-1) and $v_{\mathrm{f}}=2.0 \mathrm{~mm} / \mathrm{s}$ (setting 2-2) were carried out without changing the feed direction and without heat treatment.

A characterization of the swaged microstructure is done by metallographic analysis and microhardness measurements after the second forming step. For this purpose, small pieces of the workpieces were embedded in a polymer matrix and corroded electrolytically in a $60 \%$ aqueous nitric acid solution for $25 \mathrm{~s}$ with a voltage of $1.5 \mathrm{~V}$. Finally, hardness measurements were performed on the mechanically polished transverse section as well as longitudinal section of each embedded sample using a microhardness tester (FISCHERSCOPE H100C) with a Vickers indenter. For the transverse section the determined lines with measured points lay perpendicular to each other along the whole diameter of the samples, Figure 4a. The longitudinal 
Table 2. Forming parameters.

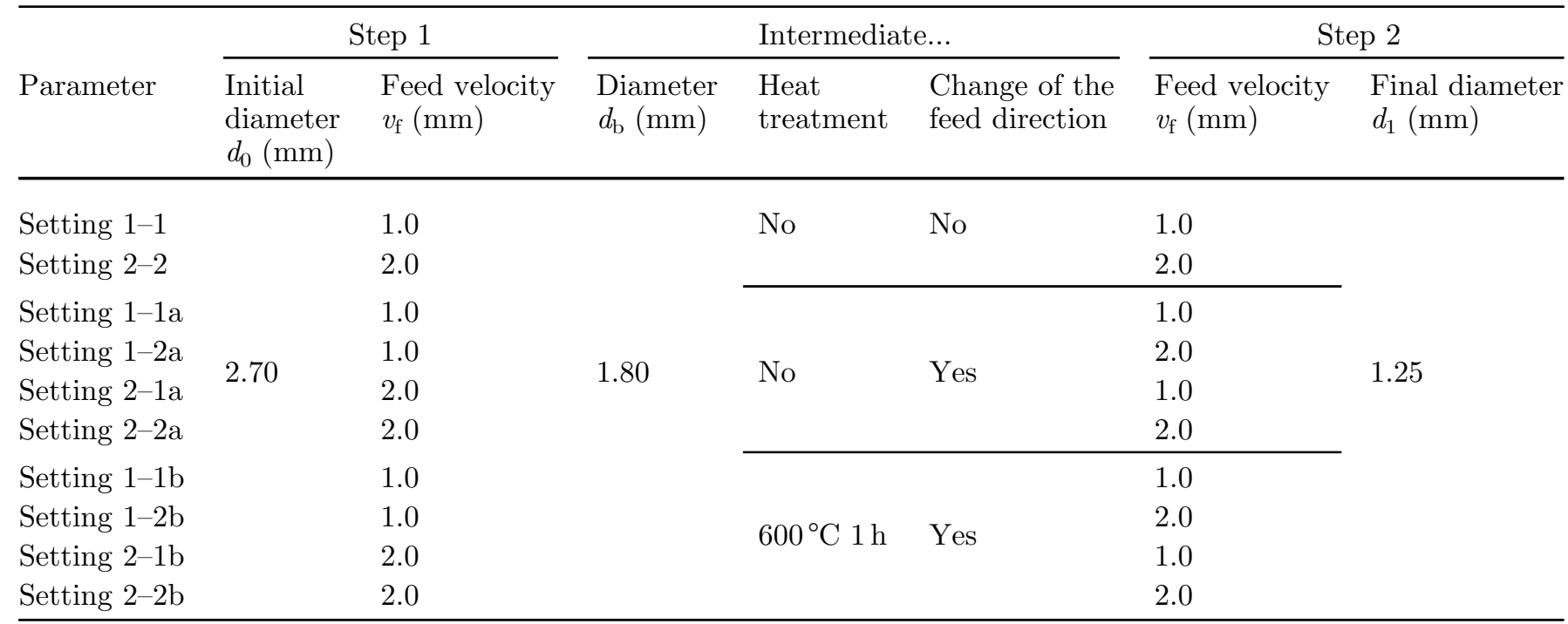

(a)

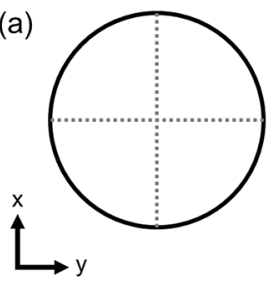

(b)

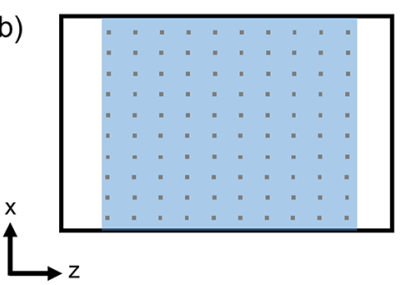

Fig. 4. Points of the hardness measurement on the (a) transverse section and (b) longitudinal section of the specimen.

section contains a rectangular area of $2.0 \times 1.2 \mathrm{~mm}^{2}$ with ten lines along $x$-coordinate direction and ten measured points along $z$-direction, Figure $4 \mathrm{~b}$.

The indentation force was $10 \mathrm{mN}$ and the duration of the penetration, holding and indenter withdrawal phases was $10 \mathrm{~s}$ for each measurement.

\section{Results and discussion}

The application of the eccentric flat swaging dies generated a significant reduction of the number and the depth of cracks on the surface of the specimens in comparison with eccentric process without EFSD [8]. In a case of two-stage formed rods without any change of direction (setting 1-1) and (setting 2-2), the crack direction was found to be identical to the rotation direction of the tools (Fig. 5). In addition, the microstructure in the transverse microsection could be divided into three zones: (I) uniformly shaped center of the workpiece; (II) severely deformed and swirled middle section of the workpiece and (III) outer section with cracks.

The increase of the feed velocity leads to more supplied material into the swaging head during each opening of the tools and thus, to a rise of the formed volume during one stroke. The process caused a changing of some properties of the workpieces after swaging, such as diameter, hardness
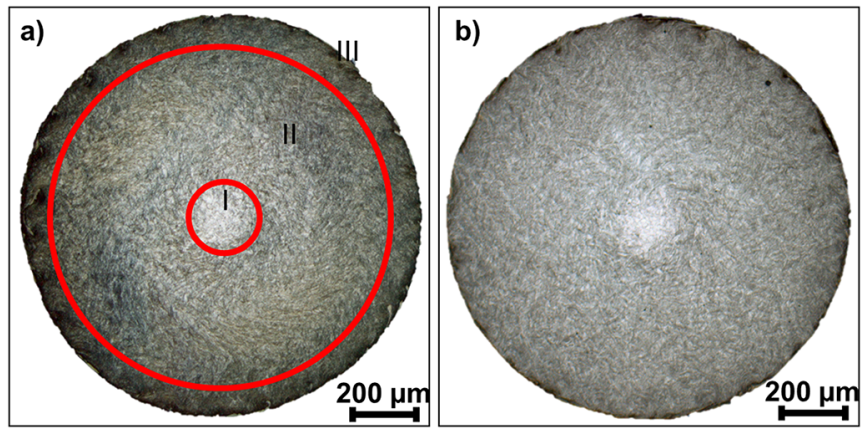

Fig. 5. Microstructure of stainless steel after forming with setting (a) 1-1 (feed velocity $v_{\mathrm{f}}=1 \mathrm{~mm} / \mathrm{s}$ ) and (b) 2-2 (feed velocity $\left.v_{\mathrm{f}}=2 \mathrm{~mm} / \mathrm{s}\right)$, both with the same feed direction in steps 1 and 2 .

and martensite content [5,10]. In the case of two-steps swaging without direction change, the microstructure of the formed parts without intermediate heat treatment was less affected by the feed velocity. However, the slow process (setting 1-1, Fig. 6a) resulted in a more pronounced inhomogeneous structure with more whirl-like pattern in zones II and III as with higher feed velocity (setting 2-2, Fig. 6d), which corresponds to the number of strokes per processing length that is higher with lower feed velocity.

With changing the direction between the two steps, the workpieces exhibited cracks after the second forming step, which were either oriented in the direction of rotation or against the direction of rotation of the tools in the second step. This is a clear evidence that some of the cracks already occurred during the first forming step (Fig. 6).

The microstructure was developed more homogeneously with direction change (Fig. 6) than without direction change (Fig. 5). It could be seen both visually by the microstructure and by the microhardness measurements.

Figure 7 shows the results of the hardness measurement of the two-stage formed workpieces with setting 2-2 without changing the feed direction between the two 


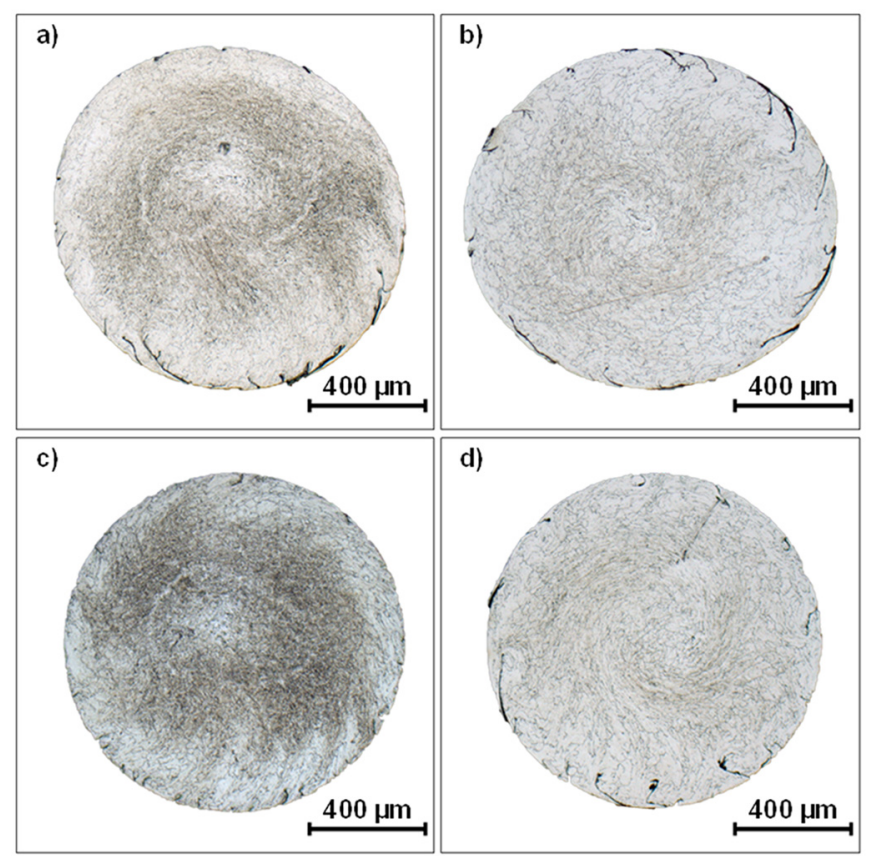

Fig. 6. Microstructure of stainless steel after forming with setting (a) 1-1a, (b) 1-2a, (c) 2-1a and (d) 2-2a with change of feed direction after step 1.

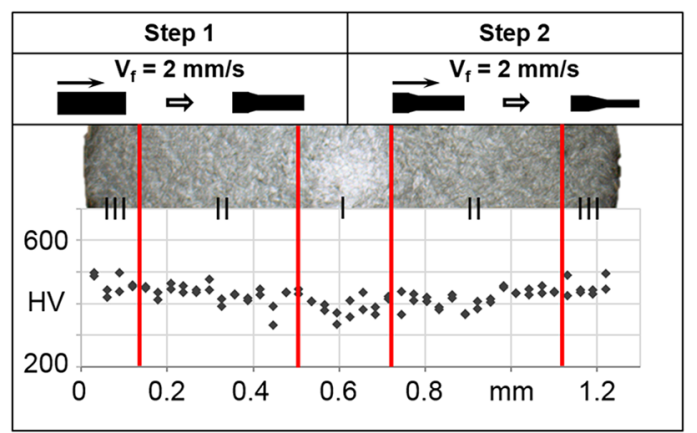

Fig. 7. Hardness tests of the transverse section after forming with setting $2-2$.

forming steps. The hardness average along the transverse microsection is about $431 \pm 28 \mathrm{HV} 0.001$ in zones II and III. In zone I, the hardness is in the range of $400 \pm 26 \mathrm{HV} 0.001$. Thus, the specimen exhibited an inhomogeneous distribution of hardness along the transverse microsection.

The measurements of the longitudinal section at setting $2-2$ are shown in Figure 8 . The average value of the hardness is $416 \pm 32 \mathrm{HV} 0.001$. This value lies within the hardness distribution of the transverse section. The shapes in $x$ - and $z$-directions show only slight differences.

By changing of the feed direction between two forming steps, a higher hardness was achieved in both transverse and longitudinal sections. The hardness of the workpiece with setting $2-2 \mathrm{a}$ is approximately $423 \pm 21 \mathrm{HV} 0.001$ in zone I and $472 \pm 23 \mathrm{HV} 0.001$ in zones II and III (Fig. 9). However, hardness values for direction change (Fig. 9) seem comparable homogenous to no direction change (Fig. 7).

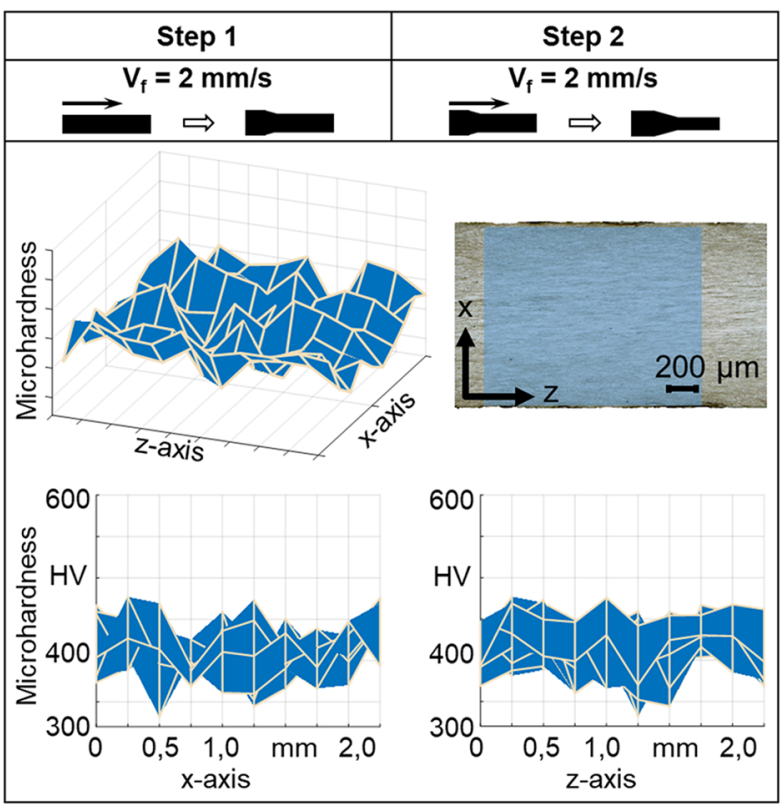

Fig. 8. Hardness tests of the longitudinal section after forming with setting $2-2$ (feed velocity $v_{\mathrm{f}}=2 \mathrm{~mm} / \mathrm{s}$ ).

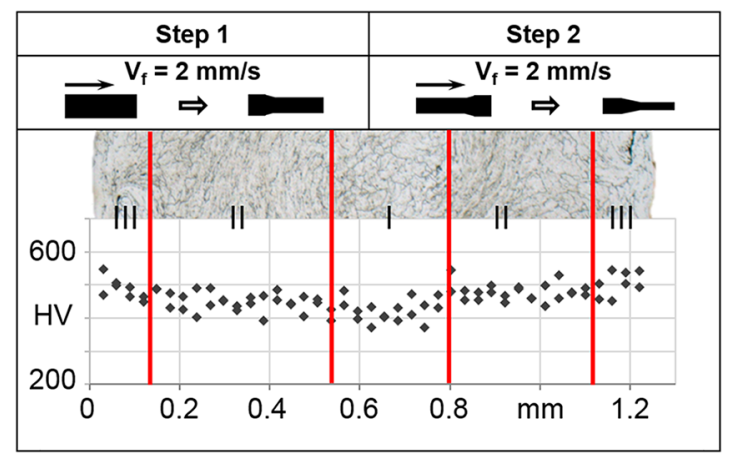

Fig. 9. Hardness tests of the transverse section after forming with setting 2-2a with change of feed direction after step 1 .

Just as for the transverse section results of the specimen swaged using setting 2-2a the hardness values for the longitudinal section for the same setting were also higher as for the workpieces formed without changing the feed direction between the two forming steps, and obtained an average value of $435 \pm 25 \mathrm{HV} 0.001$, see Figure 10 . The deviations of the measurements were smaller in comparison to that achieved with setting 2-2 and they became more homogenous at large. The shape of these measurements in $x$-direction was similar to the hardness distribution of the transverse section.

In the case of changed feed direction and additional heat treatment between two forming steps, the workpiece with setting $2-2 \mathrm{~b}$ showed a higher hardness of about $457 \pm 19 \mathrm{HV} 0.001$ in zone I and $488 \pm 38 \mathrm{HV} 0.001$ in zones II and III as for settings 2-2 and 2-2a.

However, the middle zone I was not still centric and shifted to the side. Moreover, the area of the zone III 


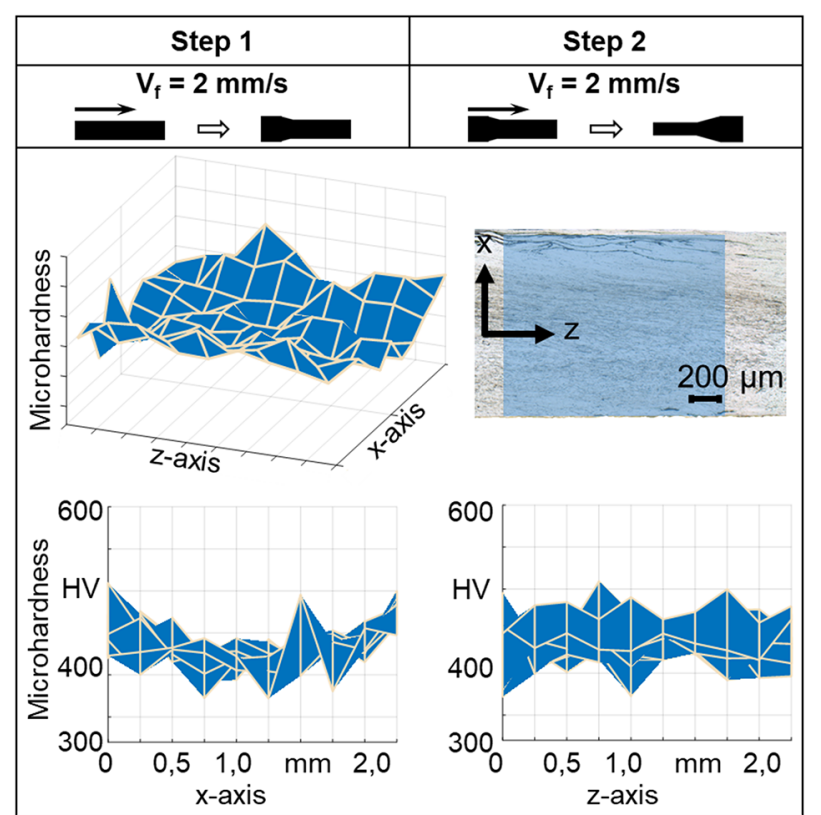

Fig. 10. Hardness tests of the longitudinal section after forming with setting $2-2 \mathrm{a}$ (feed velocity $v_{\mathrm{f}}=2 \mathrm{~mm} / \mathrm{s}$ ) with change of feed direction after step 1.

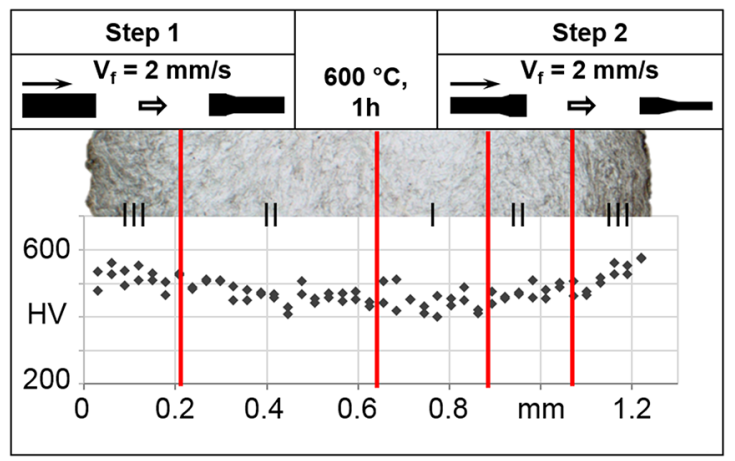

Fig. 11. Hardness tests of the transverse section after forming with setting $2-2 \mathrm{~b}$ (feed velocity $v_{\mathrm{f}}=2 \mathrm{~mm} / \mathrm{s}$ ) with change of feed direction after step 1 and heat treatment between steps 1 and 2 .

became greater due to deeper cracks on the surface. The structure for setting $2-2 \mathrm{~b}$ was more homogeneous in the middle area and had less scattering of the measured values, Figure 11. The change of the feed direction caused additional work hardening. That could be explained by three different mechanisms. The first mechanism is that the material flow changed with the different forming procedures. In consequence, the real deformation degree differed from the nominal deformation degree, which is defined only by the resulting overall change of the geometry and was the same for all experiments. The second mechanism is that the kind and direction of the stress and strain differed significantly. Hence, the hardness generation would differ especially if it was caused by martensite formation [11]. The third mechanism is that the crystallographic orientation of the constituent grains of the microstructure changed during the variation of the feed direction.

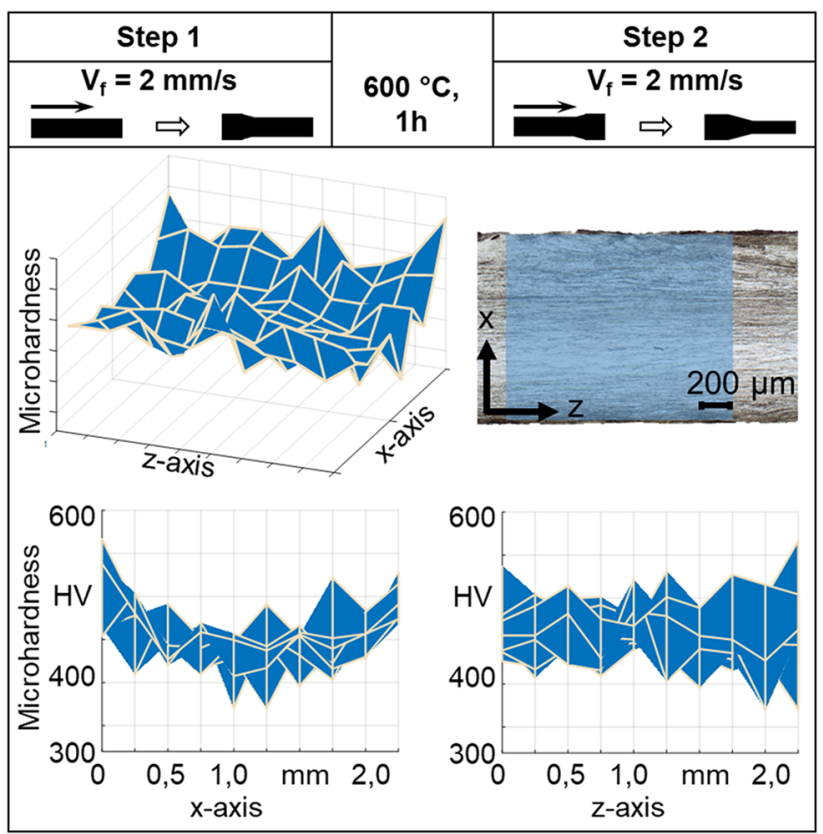

Fig. 12. Hardness tests of the longitudinal section after forming with setting $2-2 \mathrm{~b}$ with change of feed direction after step 1 and heat treatment between steps 1 and 2 .

The analysis of the samples based on setting $2-2 \mathrm{~b}$ with intermediate heat treatment provided very high values of the hardness in the longitudinal section of about $457 \pm 23 \mathrm{HV} 0.001$. The shape of the measurements in $x$ direction showed distinct patterns which is well shifted from the middle low hardness values, see Figure 12. It could be one more evidence of the non-centric zone I, see Figure 12.

The heat treatment reduced the stress and restored the material's reshaping capability. Indeed, the heat treatment after the first forming step resulted in significant degradation of the workpiece surface after the second forming step. The heat treated workpieces (setting $1-1 \mathrm{~b}, 1-2 \mathrm{~b}, 2-1 \mathrm{~b}$ and 2-2b) also featured more cracks that also penetrated significantly deeper into the structure than for the parts formed without heat treatment. Some of the heat treated samples showed parts on the surface that were torn off. Wherein, the deterioration of the surface was much stronger with the workpieces $1-1$ and $2-1$, than with the workpieces $1-2$ and $2-2$. This is due to the fact that with lower feed velocity also less volume was formed during a single stroke and the twisting effect by eccentric rotary swaging was increased. Hence, the probability increased that the cracks opened by the heat treatment and that these opened cracks could be trapped and torn between the swaging tools within the second forming step. This effect was also increased by a higher ductility of the heat-treated samples.

In the case of the lower feed velocity in step 1 then in step 2 (setting 1-2b) and the intermediate heat treatment, the microhardness featured a value of $466 \pm 23 \mathrm{HV} 0.001$ in zone I and $486 \pm 25 \mathrm{HV} 0.001$ in zone II. The value of the microhardness in zone III rose to the $466 \pm 23 \mathrm{HV} 0.001$, Figure 13. 


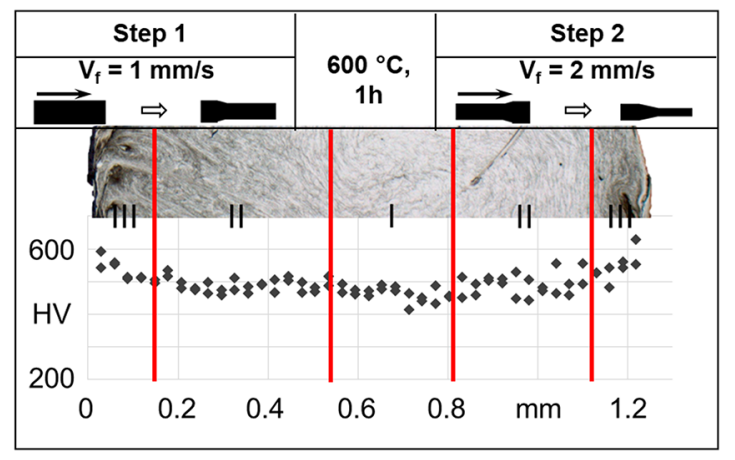

Fig. 13. Hardness tests of the transverse section after forming with setting $1-2 \mathrm{~b}$ (feed velocity $v_{\mathrm{f}}=1 \mathrm{~mm} / \mathrm{s}$ in step 1 and $v_{\mathrm{f}}=2 \mathrm{~mm} / \mathrm{s}$ in step 2) with change of feed direction after step 1 and heat treatment between steps and step 2 .

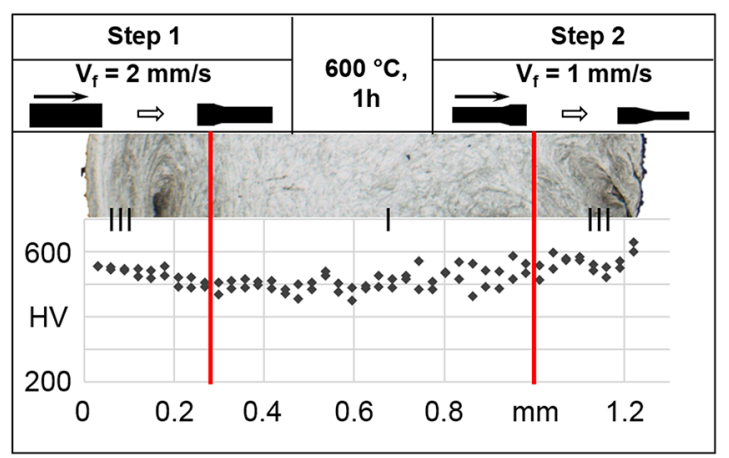

Fig. 14. Hardness tests of the transverse section after forming with setting $2-1 \mathrm{~b}$ (feed velocity $v_{\mathrm{f}}=2 \mathrm{~mm} / \mathrm{s}$ in step 1 and $v_{\mathrm{f}}=1 \mathrm{~mm} / \mathrm{s}$ in step 2) with change of feed direction after step 1 and heat treatment between steps 1 and 2 .

In contrast, with the higher feed velocity in step 1 (setting $2-1 \mathrm{~b}$ ) and the lower feed velocity in step 2 zones I and II cannot be distinguished any more. In these workpieces, the hardness changed not only between the different zones, but also within the zones: in zone III the highest value left ( $528 \pm 22 \mathrm{HV} 0.001)$ is significantly lower than the highest value right $(565 \pm 28 \mathrm{HV} 0.001)$, see Figure 14. The hardness in the middle lays in the level of $506 \pm 29 \mathrm{HV} 0.001$ with a clear tendency. In summary, the mechanical properties for the workpieces swaged with the change of feed velocity $v_{\mathrm{f}}$ between two forming steps and the intermediate heat treatment did not follow a clear pattern in the transverse section, and the definition of the zones became obsolete.

With regard to the homogeneity of the samples, the workpieces with the change of feed direction showed the best results. The combination $2-1$ a resulted in significantly less cracks than the other combinations. In the transverse section as well as in the longitudinal microsection it could be observed that the cracks had formed only at the surface of the workpieces with the forming parameters 2-1a.

In this way, the process kinematic and the intermediate heat treatment influence the microstructure and the material characteristics of the swaged parts. Individual setups give the opportunity to improve the swaging process and to produce the parts with adjustable characteristics depended on the function of the components.

\section{Conclusions}

A two-stage process of eccentric rotary swaging using EFSD was analyzed in this study. Experiments were conducted with austenitic stainless steel rods AISI304 with the initial diameter $d_{0}=2.7 \mathrm{~mm}$. The influence of changing of the feed direction and a heat treatment between the two consecutive forming steps was examined in this work. It could be stated that:

- A change of the feed direction influenced the material characteristics of the swaged workpieces. This change resulted in a higher hardness and a more homogeneous structure and, hence, changed the material properties of the swaged workpieces.

- In this way, multistage forming allows new variations of conditioning component properties through the process control.

- The heat treatment between the two forming steps with the same feed velocity $v_{\mathrm{f}}$ in combination with the change of the feed direction led to a further increase of the hardness and a lower variation of the measured values. This behavior was explained by three mechanisms: the material flow during the different forming procedures, the kind and direction of stress and strain creation and the change of the crystallographic grains orientation.

- By different feed velocity values in steps 1 and 2 and changed feed direction, the hardness gained the highest value but with an asymmetric distribution.

- The heat treatment that is commonly used in consecutive cold forming procedures was found to be harmful in multistep eccentric rotary swaging. The intermediate heat treated workpieces featured deep cracks. The cracks which occur during the first forming step become "open" by the heat treatment and negatively interacted with the tools in the following forming step.

- The multistage eccentric rotary swaging gives an opportunity to produce rods with a fine grain structure homogeneously over the transverse section.

For a better understanding of the hardening effects and the microstructure evolution during eccentric rotary swaging the intermediate heat treatment should be applied between two forming steps by the unidirectional feed of the workpiece. For future investigations the influence of the stage number will also be analyzed. The investigation of the microstructure and material properties variation by onestage forming compared to two-stage forming with the same deformation degree should give a better understanding of the material flow by microrotary swaging. It should also be discussed which adjustments of the process realization lead to highest homogeneity as well as to finest grained microstructure.

The authors would like to thank the German Research Foundation (DFG) for financial support of the project $\mathrm{T} 2$ and A2 within the collaborative research centre SFB 747 "Micro Cold Forming". 


\section{References}

1. R.Z. Valiev, R.K. Islamgaev, I.V. Aleksandrov, Bulk nanostructured materials from severe plastic deformation, Prog. Mater. Sci. 45 (2000) 103-189

2. R.J. Hellmig, Hochgradige plastische Umformung durch Equal Channel Angular Pressing (ECAP), habilitation treatise, TU Clausthal, 2008 urn:nbn:de:gbv:104-1090044

3. E. Moumi, B. Kuhfuss, Properties of alloy 304 micro parts processed by rotary swaging, in: Proceedings of 14th International Conference on Nanomanufacturing, 2014

4. B. Kuhfuss, E. Moumi, B. Clausen, J. Epp, B. Koehler, Investigation of deformation induced martensitic transformation during incremental forming of 304 stainless steel wires, Key Eng. Mater. 651-653 (2015) 645-650

5. S. Ishkina, C. Schenck, B. Kuhfuss, E. Moumi, K. Tobeck, Eccentric rotary swaging, Int. J. Precis. Eng. Man. 18 (2017) 1035-1041
6. S. Ishkina, C. Schenck, B. Kuhfuss, Conditioning of material properties by micro rotary swaging, in: Proceedings of the 18th International Conference on Material Forming, ESAFORM, 2018

7. R. von Bargen, A. von Hehl, H.-W. Zoch, Heat treatment, in: Micro Metal Forming, Springer, Switzerland, 2013, pp. 56-68

8. A. Toenjes, S. Ishkina, A. von Hehl, C. Schenck, H.-W. Zoch, B. Kuhfuss, Untersuchung der inneren Struktur von exzentrisch rundgekneteten Bauteilen aus X5CrNi18-10, 8. Kolloquium Mikroproduktion, 2017

9. A. Toenjes, S. Ishkina, A. von Hehl, C. Schenck, H.-W. Zoch, B. Kuhfuss, Multistage eccentric rotary swaging, HTM J. Heat Treat. Mater. 73 (2018) 344-351

10. B. Kuhfuss, E. Moumi, V. Piwek, Influence of the feedrate on work quality in micro rotary swaging, in: Proceedings of 3th International Conference on Micromanufacturing, ICOMM, 2008

11. A.A. Lebedev, V.V. Kosarchuk, Influence of phase transformations on the mechanical properties of austenitic stainless steels, Int. J. Plasticity 16 (2000) 749-767

Cite this article as: Svetlana Ortmann Ishkina, Anastasiya Toenjes, Christian Schenck, Axel von Hehl, Hans-Werner Zoch, Bernd Kuhfuss, Eccentric rotary swaging variants, Manufacturing Rev. 6, 15 (2019) 